\title{
Mitochondrial genome variability: the effect on cellular functional activity
}

This article was published in the following Dove Press journal:

Therapeutics and Clinical Risk Management

\author{
Aleksandrina S Volobueva' \\ Alexandra A Melnichenko² \\ Andrey V Grechko ${ }^{3}$ \\ Alexander N Orekhov ${ }^{2,4}$ \\ 'Laboratory of Gene Therapy, \\ Biocad Biotechnology Company, \\ Saint-Petersburg, Strelnya, Russia; \\ 'Laboratory of Angiopathology, \\ Institute of General Pathology and \\ Pathophysiology, Russian Academy of \\ Sciences, Moscow, Russia; ${ }^{3}$ Federal \\ Scientific Clinical Center for \\ Resuscitation and Rehabilitation, \\ Moscow, Russia; ${ }^{4}$ Institute for \\ Atherosclerosis Research, Skolkovo \\ Innovative Center, Moscow, Russia
}

Correspondence: Alexander N Orekhov Laboratory of Angiopathology, Institute of General Pathology and Pathophysiology, Russian Academy of Medical Sciences, Baltiyskaya Street 8, Moscow 125315, Russia

Tel +79031690866

Email a.h.opexob@gmail.com

\begin{abstract}
Mitochondria are the key players in cell metabolism, calcium homeostasis, and reactive oxygen species (ROS) production. Mitochondrial genome alterations are reported to be associated with numerous human disorders affecting nearly all tissues. In this review, we discuss the available information on the involvement of mitochondrial DNA (mtDNA) mutations in cell dysfunction.
\end{abstract}

Keywords: mitochondria, mutation, apoptosis, reactive oxygen species, ATP, electron transfer chain

\section{Introduction}

Mitochondria are often referred to as the "powerhouses of the cell" because they produce about $90 \%$ of cellular ATP. ${ }^{1}$ They also have some other vital functions participating in cell signaling, proliferation, and death. It is well known that mitochondrial dysfunction is implicated in a number of human diseases including, but not limited to, Leigh syndrome, ${ }^{2}$ Leber's hereditary optic neuropathy, ${ }^{3}$ mitochondrial encephalomyopathy with lactic acidosis and stroke like episodes (MELAS), ${ }^{4}$ myoclonus epilepsy with ragged red fibers (MERFF) ${ }^{5}$ Alzheimer's disease, ${ }^{6}$ Parkinson's disease, ${ }^{7}$ and different forms of cancer. ${ }^{8}$ Mitochondrial diseases affect nearly all tissues, and thus, their clinical signs are extremely various, partly because the respiratory chain malfunction leads to aberrant cell signaling. ${ }^{9}$

\section{Mitochondrial genome}

The organization of human mitochondrial genome has been extensively studied and is currently relatively well known. ${ }^{10}$ It differs substantially from the nuclear genome in almost all aspects. Mitochondrial DNA (mtDNA) of mammals is a double-stranded circular molecule $16.6 \mathrm{~kb}$ long with heavy and light strands enriched by purines and pyrimidines correspondingly. mtDNA contains 37 genes with short non-coding sequences in between and one large triple-stranded non-coding region, D-loop, containing transcriptional promoters and at least one of the proposed replication origins. mtDNA is packaged into a nucleoid by mitochondrial transcription factor A (TFAM), which is suggested to condense DNA by inducing bending and then wrapping it into compact nucleoid structures.

Replication of mtDNA occurs even in post-mitotic cells and is independent from that of the nuclear DNA. Key replisome proteins include DNA polymerase $\gamma$, mtDNA helicase Twinkle, mitochondrial single-stranded DNA-binding protein, and mitochondrial RNA polymerase (which most likely functions as the mtDNA primase). ${ }^{11}$ Despite the relatively small size of mtDNA, its replication includes diverse mechanisms such as 
rolling circle, theta, strand-displacement, strand-coupled, and ribonucleotides incorporated throughout the lagging strand (RITOLS)/bootlace. ${ }^{12}$ The robust rolling cycle mechanism is used by plants, fungi, and nematodes. Theta model of replication was studied in Drosophila and sea urchin. Mitochondrial genome in vertebrates is replicated via the following mechanisms: strand displacement, strand coupled, and RITOLS/bootlace. In the traditional strand displacement model, mtDNA replication is initiated in Oh (origin of heavy strand). Continuous and unidirectional enlargement of this D-loop proceeds for a distance of about two thirds around the circular genome. When approximately two thirds of the genome length is replicated, $\mathrm{Ol}$ (the initiation site for lagging strand synthesis) is exposed, and synthesis of the nascent L-strand proceeds in the opposite direction on the single-stranded template of the expanded D-loop. ${ }^{13}$ Holt et al ${ }^{14}$ proposed that more than one replication mechanism takes place in mammalian cells. In the proposed model named "bootlace”, pre-synthesized RNA fragments hybridize to the lagging DNA strand while leading DNA strand is being replicated. Strand-coupled model shares similarity with the theta model. Replication starts at a broad initiation zone containing the genes $c y t b$, nad5, and nad6 and proceeds bidirectionally until the D-loop region. To date, a number of mitochondrial polymerases have been described besides DNA PolG, namely, PrimPol, PolB, PolZ, PolH, and PolQ. However, their particular functions remain to be clarified. ${ }^{15}$

Previous studies revealed that endoplasmic reticulum (ER) marks the sites of mitochondria fission. ER is wrapped around mitochondria causing its constriction. ${ }^{16}$ The initial constriction then enables the assembly of the ring made of highly conserved dynamin-related protein from the family of large GTPases (Drp1 in mammals, Dnm1 in yeast). This ring is supposed to provide the mechanochemical force that completes fission of the outer and the inner mitochondrial membranes. Recently, an important discovery regarding mtDNA replication was made by simultaneously imaging mitochondria, nucleoids, and the ER network at high spatial and temporal resolution using spinning disk confocal microscopy in U2OS and Cos7 cell lines. ${ }^{17}$ It appeared that mtDNA replication is tightly coupled with its division, and these processes take place in an area of ER-mitochondrion contact. The researchers also noted that the ER tubules are required for intracellular nucleoid distribution within the cell, which is of special importance for polarized cells.

\section{Mitochondrial genome alterations}

Mitochondrial genome has a much higher mutation rate than the nuclear genome, ${ }^{18}$ which varies across species. ${ }^{19}$
Somatic and inherited mutations in mtDNA are classified into deletions and single-nucleotide replacements that can be neutral or pathological. Inherited genome variations are transmitted via the maternal line because sperm mitochondria are subjected to degradation during fertilization. With progress in high-throughput sequencing methods, more pathological mitochondrial genome mutations associated with human diseases are being reported in the Mitomap database. It has been previously hypothesized that oxidative stress was a major reason for mtDNA variability. ${ }^{20}$ According to the modern point of view, most human mtDNA somatic mutations are due to the infidelity of mtDNA polymerase encoded by the nuclear gene POLG. ${ }^{21}$ Most mtDNA deletions are rather big $(>1 \mathrm{~kb})$ and occur between two origins of replication. The exact mechanism of the deletion process remains unknown, but it can possibly be explained by polymerase stalling and mispairing during replication, as well as by topoisomerase II cleavage and other DNA double-strand breaks. ${ }^{22}$ Mitochondrial genome is polyploid, and it exists in numerous copies per cell. Therefore, a mitochondrial mutation can be homoplasmic when it is present in each DNA copy and heteroplasmic when it is present only in some copies of the mtDNA of an individual. The majority of pathogenic mutations are recessive. Studies showed that mutational load differs between tissues ${ }^{23}$ and that some mutations are tissue specific. There is a threshold (ratio of wild-type and mutant mtDNA copies) for the heteroplasmy level, above which pathological changes can be observed. ${ }^{24,25}$ The established values of this threshold are about $60 \%-90 \%$, being lower in tissues more relying on oxidative phosphorylation. ${ }^{26}$ The threshold also varies according to the type of mutations, being approximately 50\%-60\% and 70\%-90\% for mtDNA deletion and point mutations, respectively. ${ }^{27}$

\section{Mitochondrial protective pathways}

The fate of damaged mitochondria depends on two processes: mitophagy and repair. Mitophagy is dependent on receptors interacting with LC3-II on autophagosome via LC3-interacting region (LIR). Mitophagy receptors recognize molecules in the outer mitochondrial membrane (OMM), such as B-cell lymphoma 2 nineteen kilodalton interacting protein 3 (BNIP3), Nix, Bcl-2-like protein 13 (Bcl2-L-13) and FUND1, and in the inner membrane, such as cardiolipin. Several non-mitochondrial receptors recognizing both the target mitochondrion and the autophagosome include p62, NDP52, OPTN, NBR1, and TAX1BP1, which contain both LIR motif and ubiquitin-binding domain (UBD). ${ }^{28}$ Among different variants, mitophagy kinase PINK1/ubiquitin 
ligase Parkin mediated is the most thoroughly studied one. ${ }^{29}$ Normally, PINK1 kinase is transported into the mitochondria where it is ultimately degraded. In the case of disrupted membrane potential, PINK1 integrates into the mitochondrial outer membrane and recruits Parkin activating its ubiquitin ligase activity and leading to ubiquitination of multiple mitochondrial proteins. PINK1 also phosphorylates polyubiquitin chains conjugated to mitochondrial proteins by Parkin. Accumulation of polyubiquitin chains leads to the disposal of defective mitochondria in the lysosomes. Impaired clearance of dysfunctional mitochondria may act as an important component of aging. ${ }^{30}$

The other process, unfolded protein response (UPR), promotes the recovery of mitochondrial dysfunction which is mediated by the transcription factor ATFS-1. Under normal conditions, ATFS-1 is imported into mitochondria and degraded. ${ }^{31}$ During mitochondrial stress, ATFS-1 is preferentially translocated into the nucleus where it upregulates the expression of mitochondrial chaperones and various genes involved in glycolysis and amino acid catabolism pathways. ATFS-1 also accumulates within the mitochondria to repress the OXPHOS transcripts encoded by mtDNA. In summary, it provides fine tuning of OXPHOS genes expression on stress. ${ }^{32}$

UPR is generally considered as a protective mechanism, despite some concerns raised by two recent studies performed on Caenorhabditis elegans with heteroplasmic $3.1 \mathrm{~kb}$ deletion in mtDNA (variant $u a D f 5$ ). ${ }^{33,34}$ This deletion removes four protein-coding genes and seven tRNA genes and is associated with decreased basal oxygen consumption and total respiratory capacity. Interestingly, this obviously deleterious mtDNA variant is quite stable in animals. In worms with high uaDf5 levels, UPR is activated, and uaDf5 frequency decreases in the absence of UPR. Researchers proposed that this protective mechanism is responsible for maintaining the level of mutated mtDNA. Probably, UPR activation inadvertently propagates deleterious mtDNA in an attempt to recover OXPHOS activity. Both groups suggested that exploitation of homeostatic responses may represent a general strategy used for proliferation of pathogenic mtDNA mutations associated with human diseases.

The list of pathological mtDNA mutations is quite long. The main purpose of this review is to describe the consequences of the mitochondrial genome alterations at the cellular level and to provide examples of how the mitochondrial genome variability affects the cell function and causes human diseases. Nuclear genome mutations relevant for mitochondrial functions are not discussed in this review.

\section{Mitochondrial functions in the cell Mitochondria and oxidative phosphorylation}

A majority of proteins necessary for mitochondrial function are encoded by the nuclear genes and are transported from the cytoplasm. However, 13 mitochondrial genes encode proteins that are essential components of the respiratory chain: complexes (I-IV) and ATP synthase. Other mitochondrial genes are responsible for rRNA and tRNA synthesis, being used for their translation within the organelle. Proteins of the respiratory chain complexes are synthesized within the mitochondria and are located in the highly invaginated inner membrane. Oxidative phosphorylation is thoroughly explained elsewhere. ${ }^{35}$ In brief, pyruvate obtained from glycolysis is transferred from the cytoplasm into the mitochondrion, where it acquires acetyl groups from CoA. Acetyl groups are transferred to oxaloacetate and are oxidized to carbon dioxide and water in the tricarboxylic acid (TCA) cycle. Generated during glycolysis and the TCA cycle, electron-rich NADH and succinate intermediate of the TCA cycle are oxidized by the respiratory chain complexes I and II, respectively. The electrons then passed to ubiquinone, which diffuses to complex III in a reduced state in order to be re-oxidized. Respiratory chain complex III reduces cytochrome $c$, which in turn transfers the electrons to complex IV, where oxygen is reduced to water. This electron transfer leads to active pumping of hydrogen ions from the mitochondrial matrix into the intermembranous space. The resulting electrochemical gradient generated across the inner membrane facilitates proton translocation from the intermembranous space through the ATP synthase complex back into the matrix. This proton transfer is coupled with the reaction of ADP phosphorylation to generate ATP.

In addition to its importance for mitochondrial ATP synthesis, mitochondrial membrane potential $(\Delta \Psi)$ is needed for protein import into the mitochondria, respiratory substrates uptake, and ion transport across the inner mitochondrial membrane. Therefore, its maintenance is crucial for proper functioning of the mitochondrion. It is known that mutations in the respiratory chain protein genes are accompanied with decreased mitochondrial membrane potential, whereas ATP synthase dysfunction is associated with an increase in $\Delta \Psi{ }^{36}$

Direct interrelation between mtDNA variations and reduced OXPHOS system activity and lower $\Delta \Psi$ can be supported by studies discussed later. ${ }^{37-39}$ Cybrid technology allowed combining Leber hereditary optic neuropathy 
(LHON) syndrome patient platelets with homoplasmic mutation in the $M T-N D 1$ gene (m.3634A $>\mathrm{G}$, p.Ser110Gly) and mtDNA-less human osteosarcoma 143B cells. This approach allowed determining the activities of complex I, complex I + III, complex II, complex II + III, complex IV and citrate synthase along with $\Delta \Psi$. Cybrids carrying m.3634A $>\mathrm{G}$ showed decreased $(\approx 60 \%)$ complex I and I + III activity, lower cell proliferation, and decreased mitochondrial membrane potential relative to control cybrids. ${ }^{37}$

Two previously unknown heteroplasmic mtDNA mutations, namely $\mathrm{m} .8305 \mathrm{C}>\mathrm{T}$ mutation (MTTK gene) and the $\mathrm{m} .4440 \mathrm{G}>\mathrm{A}$ mutation (MTTM gene) were identified in patients suffering from myopathy. Analysis of respiratory chain enzyme activities in the skeletal muscle biopsy showed a combined defect of complexes I, III, and IV. ${ }^{38}$

In a recent study, the authors performed a comprehensive comparative analysis of the mitochondrial genome of a series of cybrids containing mitochondria of synaptosomes from mice of different ages (6 and 26 months old, representing young and old groups, respectively) using a high-resolution, highsensitivity deep sequencing method. ${ }^{39}$ Identified mtDNA point mutations were located in both D-loop (six mutations) and coding regions of $C O X-I, A T P 6, N D 5$, and $C y t B$ genes (four mutations). In total, five mutations were likely to be deleterious based on the analysis of nucleotide and amino acid conservation and the prediction of protein structural and functional change. The activity of complex I was significantly lower in the cybrids created from the old cells, likely due to the decreased complex assembly.

\section{Mitochondria as calcium depots}

Besides being the energy producing units of the cell, mitochondria also act as local calcium $\left(\mathrm{Ca}^{2+}\right)$ depots to maintain the intracellular calcium homeostasis, which is based on two continuous and opposite processes: mitochondrial calcium efflux and influx. In case of calcium overload, the mitochondrion acts as a sink for the excess calcium ions via voltage-dependent anion channel (VDAC) in the outer membrane and highly selective calcium uniporter (MCU) in the inner membrane. The uniporter is a mitochondrial membrane potential-dependent calcium selective channel located in the inner mitochondrial membrane. Calcium efflux is carried out by two mechanisms: $\mathrm{H}^{+} / \mathrm{Ca}^{2+}$ exchange and $\mathrm{Na}^{+} / \mathrm{Ca}^{2+}$ exchange. ${ }^{40}$ It is known that mitochondria operate in close conjunction with the ER in distinct sites called mitochondria-associated membranes (MAMs) that perform calcium exchange between the two compartments. ${ }^{41}$ Interestingly, proteins modulating this exchange are affected in many neurodegenerative diseases. ${ }^{42}$ There is an interrelation between the function of the electron transfer chain (ETC) and calcium ions exchange in the mitochondria. Calcium requires membrane potential for its influx, while increased concentration of calcium enhances ATP synthesis via the following enzymes: pyruvate, alpha-ketoglutarate and NADisocytrate dehydrogenases, and ATP synthase. ${ }^{43,44}$ Excess $\mathrm{Ca}^{2+}$ uptake can lead to the permeability transition pore (PTP) opening, release of cytochrome $c$, and cell death.

Pathogenic mtDNA mutations disturb the $\Delta \Psi$ and calcium uptake and signaling consequently, which was supported by the results of several recent in vitro studies. ${ }^{45,46}$ It was demonstrated using mutant cybrids that homoplasmic mutation $13565 \mathrm{C}>\mathrm{T}$ in the NADH gene (MT-ND5) leads to a significant reduction of NADH and mitochondrial membrane potential and is associated with a decrease in calcium uptake by the mitochondria. The authors suggested that in ND5 mutant ATP generated by glycolysis is required for both mitochondrial membrane potential maintenance and regulation of calcium homeostasis in the cell. ${ }^{45}$ With the help of induced pluripotent stem cells technology, a library of differentiated neural progenitor cells of patients carrying homoplasmic deleterious MT-ATP6 gene mutation (m.9185T $>\mathrm{C}$ ) was generated. This variation is common in Leigh syndrome, neuropathy, ataxia, and retinitis pigmentosa (NARP). Patient-derived neural progenitor cells with $\mathrm{m} .9185 \mathrm{~T}>\mathrm{C}$ exhibited the downregulation of genes involved in mitochondrial calcium homeostasis in contrast to mutated fibroblasts or cybrids. These cells also demonstrated the reduction of calcium-induced calcium release from intracellular stores on glutamate stimulation. ${ }^{46}$

In a yeast model, researchers investigated the relevance of two mutations in the MT-ATP6 gene (Atp6-P163S and Atp6-K90E corresponding to human MTATP6-P136S and MTATP6-K64E found in several cancer cell type) for cancer. ${ }^{47}$ Mutant cells had higher reactive oxygen species (ROS) and calcium ions level. The formation of the yeast permeability transition pore homolog (yeast permeability transition pore homolog and yeast mitochondria unspecific channel) after calcium induction was slower. The study demonstrated a link between ATP synthase function, ROS production, calcium homeostasis, and PTP formation when functioning of the porin complex in the mitochondrial outer membrane is disturbed by GFP tag fused to its component Om45p.

\section{Mitochondria and oxidative stress}

Hosting the processes of oxidative phosphorylation, mitochondria are also cellular sites of ROS production. In the respiratory chain, electrons derived from NADH or succinate 
oxidation may leak and react with $\mathrm{O}_{2}$ or other electron acceptors generating free radicals like the superoxide anion $\left(\mathrm{O}_{2}^{-}\right)$, which can be further reduced to the hydroxyl radical $\left(\mathrm{OH}^{-}\right)$and hydrogen peroxide $\left(\mathrm{H}_{2} \mathrm{O}_{2}\right)$. Respiratory chain complexes I and III are predicted to be the major sites for ROS production. ROS can damage lipids, proteins, and DNA, and several defense mechanisms against ROS exist within the cell. Superoxide is converted initially to $\mathrm{H}_{2} \mathrm{O}_{2}$ by two intracellular superoxide dismutases (SODs): $\mathrm{Cu}-\mathrm{Zn}$ SOD (SOD1) and MnSOD (SOD2), and $\mathrm{H}_{2} \mathrm{O}_{2}$ in turn can be transformed into $\mathrm{H}_{2} \mathrm{O}$ by catalase or glutathione peroxidase. There exist a number of non-enzymatic cellular ROS scavengers, for example, ascorbate, flavonoids, carotenoids, and glutathione. Thus, oxidative stress results from an imbalance between ROS generation and disposal and thereby creates oxidative damage. ${ }^{48}$

A connection between increased ROS production and mtDNA mutations has been demonstrated in numerous studies. In an early study using human cybrids generated from the fusion of enucleated MELAS or MERRF patients cells (containing the following mtDNA mutations: A3243G in the tRNALeu(UUR) and A8344G in the tRNALys) with human osteosarcoma cells devoid of mtDNA (143B), elevated ROS level was observed ${ }^{49}$ In another study on HeLa cells transfected with vectors expressing mutants of MT-ND2 gene (namely G4831A and A4605G), ROS production was markedly increased in comparison with wild-type and vector-alone controls. ${ }^{50} \mathrm{~A}$ study performed on a mouse model expressing a proofreading-deficient version (D257A) of the mtDNA polymerase $\gamma$ demonstrated increased spontaneous mtDNA mutation rates. Although muscle mitochondria in D257A mice had impaired bioenergetics associated with compromised respiration, lower ATP content, and decreased membrane potential, these effects were not accompanied by an increase in mitochondrial ROS production or oxidative damage. ${ }^{51}$

Within the cell, ROS serve as important signal transducers and actively participate in the inflammation, cancer development, and aging. ${ }^{52}$ The low levels of ROS promote proliferation, ${ }^{53}$ whereas high levels of ROS lead to apoptosis. Therefore, it was suggested that an "ROS window" is required for normal/cancer cell functions: above this level, cell death is activated, and below it, proliferation is blocked. Modifying ROS production in tumor cells is among attractive strategies of cancer treatment. ${ }^{54}$

It is still unclear whether the connection between mtDNA mutations and human aging is causative or merely correlative. A widely known free radical theory of aging has been proposed by Harman. ${ }^{55}$ Modern studies attempted to explain the links between mtDNA mutations and aging in more detail. For instance, a recent study explored the effects of C150T polymorphism in the D-loop of mtDNA, which was detected earlier in the Italian centenarians. It turned out that cybrids with haplogroups positive for polymorphism C150T have lower ROS production rate comparing to haplogroup-matched cybrids without that transition. The authors concluded that lower ROS production rate may affect the longevity. ${ }^{56}$

\section{Mitochondria and cell proliferation and death}

Mitochondria play a key role in cell death and proliferation. ETC dysfunction is often followed by impaired cell proliferation. Cybrids with the $3634 \mathrm{~A}>\mathrm{G}$ mutation in ND1 were unable to proliferate on galactose culture medium in contrast to glucose medium. This was indicative of negative effect of the mutation on cell viability, when cells were forced to obtain ATP from the OXPHOS system. Activity of complex I and complex I + III of ETC was significantly lower in mutated cybrids relative to the wild-type controls. ${ }^{37}$ In the in vitro study using cybrids carrying the heteroplasmic mutation $8839 \mathrm{G}>\mathrm{C}$ in MT-ATP6 gene associated with NARP syndrome, it was demonstrated that these cybrids were characterized by a lower cell proliferation and decreased mitochondrial membrane potential compared to isogenic wild-type cybrids..$^{57}$ The exact mechanism of ETC dysfunction influencing cell proliferation remains unclear, but several explanations have been offered. A recent study using clustered regularly interspaced short palindromic repeats-based genetic screen proposed that the key function of the ETC required for cell proliferation is to enable the synthesis of aspartate, a precursor in purine and pyrimidine synthesis. ${ }^{58}$ Results of another in vitro study performed on cells with depleted mtDNA showed that genetic reconstitution of the mitochondrial membrane potential restored ROS level and aspartate synthesis necessary for cell proliferation. Therefore, mitochondrial membrane potential is essential for cell proliferation. ${ }^{59}$

According to the current understanding, mitochondria play a central role in activating apoptotic cell death in response to cellular dysfunction. ${ }^{60}$ In vitro, high levels of $\mathrm{Ca}^{2+}$, oxidative stress, and low ATP induce the mitochondrial PTP opening, which allows the diffusion of low-molecularweight solutes across the inner membrane of the mitochondrion. Although the components of the pore are not entirely defined, ATP synthase was proposed to form its core. ${ }^{61}$ This leads to mitochondrial swelling and rupture of the OMM with the subsequent release of pro-apoptotic proteins, such 
as cytochrome $c$, SMAC/Diablo, apoptosis-inducing factor, and endonuclease $\mathrm{G}$, into the cytoplasm, where they activate downstream pathways of cell death. ${ }^{62}$ As mentioned earlier, ROS can also play the role of cell death triggers. It was shown that cybrids with $\mathrm{m} .9035 \mathrm{~T}>\mathrm{C}$ in the ATP6 gene responsible for a defective F0-ATPase had less than half of the steadystate content of ATP along with an eightfold higher ROS basal level, which led to cell death. Since these effects were compensated by the addition of antioxidants (CoQ10 and vitamin E), it was suggested that cell death was induced by abnormally high levels of ROS. ${ }^{63}$

Resistance of cancer cells to chemotherapeutics can provide a good example of interconnection between the mitochondrial genome alterations and apoptosis. Several investigations showed that mtDNA mutations could help tumor cells evading cell death in response to chemotherapy. In vitro, T6124C mutation in the cytochrome oxidase subunit 1 gene could affect the resistance of prostate cancer cells to chemotherapeutics (simvastatin). According to the analysis of caspase 3 and multiple proteins involved in cellular apoptosis, mutant cells were more resistant to simvastatin than wild-type control cells. ${ }^{64}$ The resistance of cybrids generated from human pancreatic cancer cell lines CFPAC-1 and CFPAC-2 to cancer therapeutics has been studied. The first line had four mutations (T10970C in the ND4 gene, T8696C, T9070G in the ATP-6 gene, and A2905G in the $16 \mathrm{~S} r R N A$ gene), the second line harbored two mutations (G6267A in the COI gene and G10176A in the ND3 gene). Cybrids from healthy subjects' mitochondria and EB8 cells were used as controls. On exposure to staurosporine, 5-fluorouracil, and cisplatin, mutant cybrids exhibited potential anti-apoptotic activity. This effect was further observed in vivo in mice bearing tumors generated from the above-mentioned cybrids. ${ }^{65}$

Sarcopenia, age-induced loss of skeletal muscle mass and function resulting from fiber atrophy and loss of muscular fibers, is another example of cell death associated with mitochondrial ETC dysfunction. Studies on rat models demonstrated that regions of muscular fibers with deletions in genes of ETC proteins were positive for markers of cell death. ${ }^{66,67}$

\section{Mitochondria-associated diseases and conditions}

Mitochondrial ETC malfunctions have dramatic consequences for the cell, including ATP and calcium uptake decrease, as well as increased ROS production. These effects can potentially lead to cell dysfunction or death. The list of mitochondria-associated disorders is quite long: from rare syndromes to cancer and neurodegenerative diseases (according to the United Mitochondrial Disease Foundation [UMDF], http://www.umdf.org). For some of these conditions, the exact mechanisms of pathogenesis remain to be elucidated, although it is known that most of them are characterized by insufficient energy supply, calcium dyshomeostasis, or ROS accumulation.

A mechanistic study using induced pluripotent stem cells was performed to clarify the molecular pathological mechanism of action of mutation 5541C $>$ T associated with MELAS. By using iPSC-based strategy, namely, diseaserelevant iPSCs derived from myoblasts of the patient, it was demonstrated that $\mathrm{m} .5541 \mathrm{C}>\mathrm{T}$ inhibited complex IV formation. That in turn impaired mitochondrial biogenesis and bioenergetics leading to decreased ATP level, increased oxidative stress level, and damaged membrane potential. ${ }^{68}$

It was suggested that some cell types in mtDNA patients should function relatively normally until there is an increased ATP demand, but highly energy-demanding cells, such as myocytes and neurons, can be severely affected. Mitochondrial myopathy (MM) can affect literally any muscle; therefore, it has various clinical features: from myalgia to spontaneous abortion. Subsarcolemmal accumulation of mitochondria to compensate the lack of ATP production is a classic feature of MM, which can be detected using histochemistry methods (so-called ragged blue fibers) or the Gomori trichrome staining (so-called ragged red fibers $[R R F]) .{ }^{69}$ Decreased energy production resulting from ETC proteins mutations can have fatal consequences in cardiac myofibers, highly depending on energy for contraction. It was estimated that the heart consumes approximately $8 \%$ of total body energy. Cardiomyopathy is one of the causes of heart failure, which, despite the enormous cost of medical healthcare, still remains the leading cause of mortality among the elderly. ${ }^{70}$

ATP production and $\mathrm{Ca}^{2+}$ homeostasis are vital for adequate neuron function. ATP is required for the maintenance of the $\mathrm{Na}^{+} / \mathrm{K}^{+}$gradient crucial for impulse transmission. Moreover, ATP functions as an extracellular signaling molecule. The lack of energy in the brain due to mutations in the ETC genes is often manifested by seizures, which are a clinical sign of mitochondrial epilepsy. ${ }^{71}$ Calcium fluxes play critical roles in the regulation of neurite outgrowth and synaptogenesis, synaptic transmission and plasticity, and neurons survival. According to the so-called calcium hypothesis of neurodegenerative diseases, atrophic and degenerative processes in the neurons in Alzeimer's disease, Parkinson's 
disease, and amyotrophic lateral sclerosis are accompanied by alterations in calcium homeostasis. ${ }^{72}$ Neurological disorders are also characterized by higher levels of ROS.

The Mitomap database contains various pathological mutations in the mitochondrial genome associated with neurological disorders like Alzeimer's disease, LHON, MELAS, MERRF, NARP, Leigh syndrome, and KearnsSayre syndrome. Each disorder is presented with several mtDNA alterations. For example, LHON typically manifested as bilateral, painless, subacute visual failure, with $90 \%$ of cases being due to one of the three mutations in NADH dehydrogenase complex subunit genes: $\mathrm{m} .3460 \mathrm{G}>\mathrm{A}$, m.11778G $>$ A, and m.14484T $>$ C. The first pathogenic variant is associated with the most severe phenotype. ${ }^{73}$

In addition to the disorders described above, accumulating experimental data indicate the involvement of mitochondrial dysfunction and mutations in aging and cancer. It is known that mtDNA mutations accumulate with aging and contribute to cell death in older patients. Impaired OXPHOS function promotes invasion, metastases, and apoptosis resistance of tumor cells. Detailed description of mitochondria participation in aging and tumor development and progression is beyond the scope of the present review and can be found elsewhere. ${ }^{74,75}$

\section{Conclusion}

Mitochondria play several important roles in cell functions, and mitochondrial dysfunction affects cell activity, proliferation, and death. Even though it is obvious that alterations in mitochondrial genes are involved in human diseases, there is currently a lack of understanding of the exact underlying pathological mechanisms. Further mechanistic studies that can clarify exact molecular steps in pathogenesis of mitochondria-associated disorders are required.

\section{Acknowledgments}

This study was supported by the Russian Science Foundation (grant number 14-14-01038).

\section{Disclosure}

ASV is employed by Biocad Biotechnology Company. The authors report no other conflicts of interest in this work.

\section{References}

1. Schon EA, DiMauro S, Hirano M. Human mitochondrial DNA: roles of inherited and somatic mutations. Nat Rev Genet. 2012;13(12): 878-890.

2. Makino M, Horai S, Goto Y, Nonaka I. Mitochondrial DNA mutations in Leigh syndrome and their phylogenetic implications. J Hum Genet. 2000;45:69-75.
3. Wallace DC, Singh G, Lott MT, et al. Mitochondrial DNA mutation associated with Leber's hereditary optic neuropathy. Science. 1988; 242(4884):1427-1430.

4. Goto Y, Nonaka I, Horai S. A mutation in the tRNA(Leu)(UUR) gene associated with the MELAS subgroup of mitochondrial encephalomyopathies. Nature. 1990;348(6302):651-653.

5. Silvestri G, Moraes CT, Shanske S, Oh SJ, DiMauro S. A new mtDNA mutation in the tRNA(Lys) gene associated with myoclonic epilepsy and ragged-red fibers (MERF). Am J Hum Genet. 1992;51(6):1213-1217.

6. Hutchin TP, Heath PR, Pearson RC, Sinclair AJ. Mitochondrial DNA mutations in Alzheimer's disease. Biochem Biophys Res Commun. 1997;241(2):221-225

7. Bender A, Krishnan KJ, Morris CM, et al. High levels of mitochondrial DNA deletions in substantia nigra neurons in aging and Parkinson disease. Nat Genet. 2006;38:515-517.

8. Chatterjee A, Mambo E, Sidransky D. Mitochondrial DNA mutations in human cancer. Oncogene. 2006;25:4663-4674.

9. Taylor RW, Turnbull DM. Mitochondrial DNA mutations in human disease. Nat Rev Genet. 2005;6(5):389-402.

10. Taanman J-W. The mitochondrial genome: structure, transcription, translation and replication. Biochem Biophys Acta. 1999;1410(2): $103-123$.

11. McKinney E, Oliveira M. Replicating animal mitochondrial DNA. Genet Mol Biol. 2013;36(3):308-315.

12. Ciesielski L, Oliveira M, Kaguni L. Animal mitochondrial DNA replication. Enzymes. 2016;39:255-292.

13. Brown T, Cecconi C, Tkachuk A, Bustamante C, Clayton D. Replication of mammalian mitochondrial DNA occurs by strand displacement with alternative light-strand origins. Genes Dev. 2005;19(20):2466-2476.

14. Holt I, Lorimer H, Jacobs H. Coupled leading- and lagging-strand synthesis of mammalian mitochondrial DNA. Cell. 2000;100(5): 515-524.

15. Krasich R, Copeland W. DNA polymerases in the mitochondria: a critical review of the evidence. Front Biosci (Landmark Ed). 2017;22:692-709.

16. Friedman J, Lackner L, West M, DiBenedetto J, Nunnari J, Voeltz G. ER tubules mark sites of mitochondrial division. Science. 2011;334(6054): $358-362$.

17. Lewis S, Uchiyama L, Nunnari J. ER-mitochondria contacts couple mtDNA synthesis with mitochondrial division in human cells. Science. 2016;353(6296):aaf5549.

18. Haag-Liautard C, Coffey N, Houle D, Lynch M, Charlesworth B, Keightley PD. Direct estimation of the mitochondrial DNA mutation rate in Drosophila melanogaster. PLoS Biol. 2008;6(8):e204.

19. Nabholz B, Glémin S, Galtier N. The erratic mitochondrial clock: variations of mutation rate, not population size, affect mtDNA diversity across birds and mammals. BMC Evol Biol. 2009;10(9):54.

20. Harman D. The biologic clock: the mitochondria? J Am Geriatr Soc. 1972;20(4):145-147.

21. Itsara LS, Kennedy SR, Fox EJ, et al. Oxidative stress is not a major contributor to somatic mitochondrial DNA mutations. PLoS Genet. 2014;10(2):e1003974.

22. Kujoth GC, Bradshaw PC, Haroon S, Prolla TA. The role of mitochondrial DNA mutations in mammalian aging. PLoS Genet. 2007; 3(2):e24.

23. Tam EW, Feigenbaum A, Addis JB, et al. A novel mitochondrial DNA mutation in COX1 leads to strokes, seizures, and lactic acidosis. Neuropediatrics. 2008;39(6):328-334.

24. Frederiksen AL, Andersen PH, Kyvik KO, Jeppesen TD, Vissing J, Schwartz M. Tissue specific distribution of the $3243 \mathrm{~A} \rightarrow \mathrm{G}$ mtDNA mutation. J Med Genet. 2006;43(8):671-677.

25. Li M, Schröder R, Ni S, Madea B, Stoneking M. Extensive tissuerelated and allele-related mtDNA heteroplasmy suggests positive selection for somatic mutations. Proc Natl Acad Sci US A. 2015;112(8): 2491-2496.

26. Tuppen HA, Blakely EL, Turnbull DM, Taylor RW. Mitochondrial DNA mutations and human disease. Biochim Biophys Acta. 2010; 1797(2):113-128. 
27. Casoli T, Spazzafumo L, Di Stefano G, Conti F. Role of diffuse lowlevel heteroplasmy of mitochondrial DNA in Alzheimer's disease neurodegeneration. Front Aging Neurosci. 2015;7:142.

28. Martinez-Vicente M. Neuronal mitophagy in neurodegenerative diseases. Front Mol Neurosci. 2017;10:64.

29. Pellegrino MW, Haynes CM. Mitophagy and the mitochondrial unfolded protein response in neurodegeneration and bacterial infection. $B M C$ Biol. 2015;13:22.

30. Diot A, Morten K, Poulton J. Mitophagy plays a central role in mitochondrial ageing. Mamm Genome. 2016;27:381-395.

31. Nargund AM, Pellegrino MW, Fiorese CJ, Baker BM, Haynes CM. Mitochondrial import efficiency of ATFS-1 regulates mitochondrial UPR activation. Science. 2012;337(6094):587-590.

32. Nargund AM, Fiorese CJ, Pellegrino MW, Deng P, Haynes CM. Mitochondrial and nuclear accumulation of the transcription factor ATFS-1 promotes OXPHOS recovery during the UPR(mt). Mol Cell. 2015;58(1):123-133.

33. Gitschlag BL, Kirby CS, Samuels DC, Gangula RD, Mallal SA, Patel MR. Homeostatic responses regulate selfish mitochondrial genome dynamics in C. elegans. Cell Metab. 2016;24(1):91-103.

34. Lin YF, Schulz AM, Pellegrino MW, Lu Y, Shaham S, Haynes CM. Maintenance and propagation of a deleterious mitochondrial genome by the mitochondrial unfolded protein response. Nature. 2016;533(7603): 416-419.

35. Rich PR, Maréchal A. The mitochondrial respiratory chain. Essays Biochem. 2010;47:1-23.

36. Szczepanowska J, Malinska D, Wieckowski MR, Duszynski J. Effect of mtDNA point mutations on cellular bioenergetics. Biochim Biophys Acta. 2012;1817(10):1740-1746.

37. Carreño-Gago L, Gamez J, Cámara Y, et al. Identification and characterization of the novel point mutation $\mathrm{m} .3634 \mathrm{~A}>\mathrm{G}$ in the mitochondrial MT-ND1 gene associated with LHON syndrome. Biochim Biophys Acta. 2017;1863(1):182-187.

38. Scarpelli M, Carreño-Gago L, Russignan A, et al. Identification and characterization of the novel $\mathrm{m} .8305 \mathrm{c}>\mathrm{T}$ MTTK and $\mathrm{m} .4440 \mathrm{~g}>\mathrm{a}$ MTTM gene mutations causing mitochondrial myopathies. Neuromusc Disord. Epub 2017 Oct 31.

39. Li H, Shen L, Hu P, et al. Aging-associated mitochondrial DNA mutations alter oxidative phosphorylation machinery and cause mitochondrial dysfunctions. Biochim Biophys Acta. 2017;1863(9):2266-2273.

40. Santulli G. Advances in Experimental Medicine and Biology. Springer International Publishing; 2017.

41. Szymański J, Janikiewicz J, Michalska B, et al. Interaction of mitochondria with the endoplasmic reticulum and plasma membrane in calcium homeostasis, lipid trafficking and mitochondrial structure. Int J Mol Sci. 2017;18(7):1576.

42. Krols M, van Isterdael G, Asselbergh B, et al. Mitochondria-associated membranes as hubs for neurodegeneration. Acta Neuropathol. 2016;131(4):505-523.

43. McInnes J. Mitochondrial-associated metabolic disorders. Nutr Metab (Lond). 2013;10(1):63.

44. Tarasov AI, Griffiths EJ, Rutter GA. Regulation of ATP production by mitochondrial $\mathrm{Ca}(2+)$. Cell Calcium. 2012;52(1):28-35.

45. McKenzie M, Duchen MR. Impaired cellular bioenergetics causes mitochondrial calcium handling defects in MT-ND5 mutant cybrids. PLoS One. 2016;11(4):e0154371.

46. Lorenz C, Lesimple P, Bukowiecki R, et al. Human iPSC-derived neural progenitors are an effective drug discovery model for neurological mtDNA disorders. Cell Stem Cell. 2017;20(5):659.e-674.e.

47. Niedzwiecka K, Tisi R, Penna S, Lichocka M, Plochocka D, Kucharczyk R. Two mutations in mitochondrial ATP6 gene of ATP synthase, related to human cancer, affect ROS, calcium homeostasis and mitochondrial permeability transition in yeast. Biochim Biophys Acta. 2018; 1865(1):117-131.

48. Lagouge M, Larsson NG. The role of mitochondrial DNA mutations and free radicals in disease and ageing. $J$ Intern Med. 2013;273(6): $529-543$.
49. Vives-Bauza C, Gonzalo R, Manfredi G, Garcia-Arumi E, Andreu AL. Enhanced ROS production and antioxidant defenses in cybrids harboring mutations in mtDNA. Neurosci Lett. 2005;391(3):136-141.

50. Zhou S, Kachhap S, Sun W, et al. Frequency and phenotypic implications of mitochondrial DNA mutations in human squamous cell cancers of the head and neck. Proc Natl Acad Sci U S A. 2007;104(18):7540-7545.

51. Hiona A, Sanz A, Kujoth GC, et al. Mitochondrial DNA mutations induce mitochondrial dysfunction, apoptosis and sarcopenia in skeletal muscle of mitochondrial DNA mutator mice. PLoS One. 2010;5(7): e11468.

52. Sullivan LB, Chandel NS. Mitochondrial reactive oxygen species and cancer. Cancer Metab. 2014;2:17.

53. Liu YW, Sakaeda T, Takara K, et al. Effects of reactive oxygen species on cell proliferation and death in HeLa cells and its MDR1-overexpressing derivative cell line. Bio Pharm Bull. 2003;26(2):278-281.

54. Panieri E, Santoro MM. ROS homeostasis and metabolism: a dangerous liason in cancer cells. Cell Death Dis. 2016;7(6):e2253.

55. Harmann D. Aging: a theory based on free radical and radiation chemistry. J Gerontol. 1956;11(3):298-300.

56. Chen A, Raule N, Chomyn A, Attardi G. Decreased reactive oxygen species production in cells with mitochondrial haplogroups associated with longevity. PLoS One. 2012;7(10):e46473.

57. Blanco-Grau A, Bonaventura-Ibars I, Coll-Cantí J, et al. Identification and biochemical characterization of the novel mutation $\mathrm{m} .8839 \mathrm{G}>\mathrm{C}$ in the mitochondrial ATP6 gene associated with NARP syndrome. Genes Brain Behav. 2013;12(8):812-820.

58. Birsoy K, Wang T, Chen WW, Freinkman E, Abu-Remaileh M, Sabatini DM. An essential role of the mitochondrial electron transport chain in cell proliferation is to enable aspartate synthesis. Cell. 2015; 162(3):540-551.

59. Martínez-Reyes I, Diebold LP, Kong H, et al. TCA cycle and mitochondrial membrane potential are necessary for diverse biological functions. Mol Cell. 2016;61(2):199-209.

60. Vakifahmetoglu-Norberg H, Ouchida AT, Norberg E. The role of mitochondria in metabolism and cell death. Biochem Biophys Res Commun. 2017;482(3):426-431.

61. Giorgio V, von Stockum S, Antoniel M, et al. Dimers of mitochondrial ATP synthase form the permeability transition pore. Proc Natl Acad Sci U S A. 2013;110:5887-5892.

62. James AM, Murphy J. How mitochondria affect cell function. J Biomed Sci. 2002;9:475-487.

63. Sikorska M, Sandhu JK, Simon DK, et al. Identification of ataxiaassociated mtDNA mutations (m.4052T $>\mathrm{C}$ and $\mathrm{m} .9035 \mathrm{~T}>\mathrm{C}$ ) and evaluation of their pathogenicity in transmitochondrial cybrids. Muscle Nerve. 2009;40(3):381-394.

64. Sun Q, Arnold RS, Sun CQ, Petros JA. A mitochondrial DNA mutation influences the apoptotic effect of statins on prostate cancer. Prostate. 2015;75(16):1916-1925.

65. Mizutani S, Miyato Y, Shidara Y, et al. Mutations in the mitochondrial genome confer resistance of cancer cells to anticancer drugs. Cancer Sci. 2009;100(9):1680-1687.

66. Cheema N, Herbst A, McKenzie D, Aiken JM. Apoptosis and necrosis mediate skeletal muscle fiber loss in age - induced mitochondrial enzymatic abnormalities. Aging Cell. 2015;14(6):1085-1093.

67. Herbst A, Wanagat J, Cheema N, Widjaja K, McKenzie D, Aiken JM. Latent mitochondrial DNA deletion mutations drive muscle fiber loss at old age. Aging Cell. 2016;15(6):1132-1139.

68. Hatakeyama H, Katayama A, Komaki H, Nishino I, Goto Y. Molecular pathomechanisms and cell-type-specific disease phenotypes of MELAS caused by mutant mitochondrial tRNA(Trp). Acta Neuropathol Commun. 2015;22(3):52.

69. Pfeffer G, Chinnery PF. Diagnosis and treatment of mitochondrial myopathies. Ann Med. 2013;45(1):4-16.

70. Brown DA, Perry JB, Allen ME, et al. Mitochondrial function as a therapeutic target in heart failure. Nat Rev Cardiol. 2017;14:238-250.

71. Rahman S. Mitochondrial disease and epilepsy. Dev Med Child Neurol. 2012;54(5):397-406. 
72. Bezprozvanny I. Calcium signaling and neurodegeneration. Acta Naturae. 2010;2(1):72-82.

73. Yu-Wai-Man PF, Chinnery PF. Leber hereditary optic neuropathy. In: Adam MP, Ardinger HH, Pagon RA, et al, editors. SourceGeneReviews ${ }^{\mathbb{R}}$ [Internet]. Seattle (WA): University of Washington, Seattle; 1993-2018.
74. van Gisbergen MW, Voets AM, Starmans MH, et al. How do changes in the mtDNA and mitochondrial dysfunction influence cancer and cancer therapy? Challenges, opportunities and models. Mutat Res Rev Mutat Res. 2015;764:16-30.

75. Kauppila TES, Kauppila JHK, Larsson NG. Mammalian mitochondria and aging: an update. Cell Metab. 2017;25(1):57-71.

\section{Publish your work in this journal}

Therapeutics and Clinical Risk Management is an international, peerreviewed journal of clinical therapeutics and risk management, focusing on concise rapid reporting of clinical studies in all therapeutic areas, outcomes, safety, and programs for the effective, safe, and sustained use of medicines. This journal is indexed on PubMed Central, CAS,
EMBase, Scopus and the Elsevier Bibliographic databases. The manuscript management system is completely online and includes a very quick and fair peer-review system, which is all easy to use. Visit http://www.dovepress.com/testimonials.php to read real quotes from published authors.

Submit your manuscript here: http://www.dovepress.com/therapeutics-and-clinical-risk-management-journal 